\title{
Social support reduces residual dizziness after canalith repositioning procedure in benign paroxysmal positional vertigo.
}

\author{
Zuowei Duan\#, Changbiao Fu", Tieyu Tang, Bin Liu, Jiangbing Liu, Bin Chen, Xinjiang Zhang* \\ Department of Neurology, the First People's Hospital of Yangzhou, Yangzhou, PR China \\ \#These authors contributed equally to this work
}

\begin{abstract}
Objective: Benign Paroxysmal Positional Vertigo (BPPV) is the most common cause for peripheral vestibular vertigo. However, some patients still complain Residual Dizziness (RD) even after a successful Canalith Repositioning Procedure (CRP). This study was to explore the features of RD and its association with social support.

Subjects and methods: Between January 2015 and January 2016, 258 patients with BPPV were prospectively recruited in this study. The presence of RD was investigated after a successful CRP. Social Support Rating Scale (SSRS) and Dizziness Handicap Inventory (DHI) were adopted to evaluate the status of social support and the self-perceived handicapping effects of RD respectively.

Results: Of them, 105 patients complained of RD after successful CRP. Univariate analysis indicated that the age, female sex, the length from BPPV onset to CRP and DHI score and SSRS score were significantly different between patients with RD and those without. After adjusting the confounding factors, multivariate logistic regression analysis suggested that the SSRS total score (OR 0.923, 95\% CI 0.889-0.958, $P=0.000)$ and the 3 subdomains of SSRS (subject support (OR 0.912, 95\% CI 0.869-0.956, $\mathrm{P}=\mathbf{0 . 0 0 0}$ ), objective support $(\mathrm{OR} \mathbf{0 . 8 8 9}, 95 \%$ CI $0.799-0.989, P=0.031)$, and degree of social utilization (OR $0.825,95 \%$ CI $0.716-0.951, P=0.008)$ ) were independently associated with the presence of $R D$ after CRP. In conclusion, Residual dizziness is a common condition in patients with BPPV after CRP.

Conclusion: Our study indicates that social support could help in reducing the incidence of RD in patients with BPPV after successfully CRP and returning to daily life more rapidly and comfortably.
\end{abstract}

Keywords: Benign paroxysmal positional vertigo, Residual dizziness, Canalith repositioning procedure, Social support, Dizziness handicap inventory.

\section{Introduction}

Benign Paroxysmal Positional Vertigo (BPPV), characterized by short repeated episodes of mild to intense vertigo triggered by changes in head position, and accompanied by imbalance and nausea, is the most common cause for peripheral vestibular vertigo $[1,2]$. The suggested pathophysiology is that otoconia derived from the utricular macula moves to the semicircular canal [3-5]. The movement of the otoconial matter due to gravity causes the flow of endolymph, which consequently induce vertigo and nystagmu. Currently, the Canalith Repositioning Procedure (CRP), the main treatment of BPPV proposed by Epley, is the most widely prevalent as a first-line treatment of BPPV and has been found to be safe and effective [3-7]. However, some patients still complain residual symptoms even after disappearance of typical vertigo and nystagmus following a successful CRP. These residual symptoms, also called Residual Dizziness (RD), include nonpositional, sustained imbalance of variable duration, and its pathogenesis is still poorly elucidated [8-10]. Due to these unresolved symptoms, some psychological and vegetative symptoms may manifested as anxiety, depression, fear and insomnia, which lead to a significant deterioration of the patient's functioning and reduced quality of life. Similarly, RD may also have some impact on their family's quality of life that might provide long-term day-to-day care.

With the change of health concept and medical pattern, more and more attention has been paid on the influence of psychosocial factors, such as mental status, personality and behavior, on physical and psychological health. Social support, a psychosocial factor that might help individuals in completing their treatment and coping with psychological distress, adopting healthy behaviors and improving their health, has attracted increasing attention [11-13]. Previous studies had shown that social support has a positive impact on stress relief, treatment compliance, and quality of life in patients with cardio-cerebral vascular diseases [14-17]. Although efforts have been made to evaluate the mental status of RD patients, previous studies have not assessed the amount of social support 
received by these patients $[8,18]$. Therefore, this study was to explore the amount of social support and its association with the development of RD after successful CRP in patients with benign paroxysmal positional vertigo.

\section{Subjects and Methods}

\section{Patient selection}

Between January 2015 and January 2016, 258 patients with BPPV were prospectively recruited in this study. The criteria for inclusion in the study were: 1 . no history of vertigo and vestibular disorders; 2. the first diagnosis of BPPV; 3. absence of nystagmus and positional vertigo after CRP, 4.the patient should be able to accept follow up. Exclusion criteria 1 . concomitant neurological disorders documented clinically or by imaging, patients with migraine and those in drug treatment for depression, with high levels of anxiety and a history of panic attacks; 2. Central disease (including central vertigo), terminal disease, medical or surgical conditions; 3. Vascular disorders or cranial traumas; 4. Those patients with age $>80 \mathrm{y}$ that unsuitable for CRP were also excluded from the study. The study was approved by Ethics Committees of the First People's Hospital of Yangzhou and written informed consent was obtained from each patient.

\section{Diagnosis and treatment}

The diagnosis of BPPV was based on the following criteria: 1 . symptoms compatible with BPPV (episodes of transient attacks of rotational vertigo induced by sudden head positional changes without auditory symptoms); 2 . Positional vertigo and positional nystagmus provoked by Dix-Hallpike maneuver. The Dix-Hallpike test was considered positive if nystagmus was recorded with appropriate positioning, latency, duration, and fatigue, and reversed when the patient resumed a sitting position [19]. With the affected ear down, geotropic torsional nystagmus (i.e., the upper poles of the eyes beating to the lowermost ear) occurs with an up-beating component for the Posterior Canal (PC) and a down-beating component for the Anterior Canal (AC). Horizontal Canal (HC) BPPV was diagnosed by horizontal direction-changing positional nystagmus concurrent with vertigo elicited by the supine headturning test. The patients were treated with different CRP according to the type of BPPV. Epley's CRP was used for patients with PC-BPPV. And the reverse Epley maneuver and barbecue rotation were used for patients with $\mathrm{AC}$ and $\mathrm{HC}$ BPPV, respectively [19-21]. The maneuvers in this study were done by Dr. Bin Liu and were performed several times until the disappearance of positional vertigo and positional nystagmus (i.e., until the achievement of a successful CRP). The success was determined 2 or $3 \mathrm{~d}$ after the treatment based on the result of the positioning test. Once a patient was determined to be a candidate for enrolment, patient was given a full explanation of the research protocol and was asked to return for a posttreatment visit $3 \mathrm{~d}$ later. During the first visit after the successful CRP, we collected information on the presence of any residual dizziness and its characteristics. RD was defined as feeling of unsteadiness and/or light-headedness and/or dizziness in the absence of true vertigo and nystagmus8. Then, patients reporting $\mathrm{RD}$ were evaluated every $3 \mathrm{~d}$ until the residual symptoms disappeared. The duration of RD which was from successful repositioning to complete resolution of any residual dizziness was precisely quantified.

\section{SSRS and DHI}

During the first visit after the successful CRP, the Social Support Rating Scale (SSRS) and Dizziness Handicap Inventory (DHI) were adopted to evaluate the status of social support and the self-perceived handicapping effects of RD respectively. The SSRS consists of 10 items and three dimensions, namely objective support, subjective support and support utilization, which has been demonstrated to show high reliability and validity on wide range of Chinese populations $[23,24]$. The dimension of objective support has 3 items and reflects the degree of actual support that an individual received in the past. The dimension of subjective support contains 4 items and reflects the perceived interpersonal network that an individual can count on. The dimension of support utilization includes 3 items which refers to the pattern of behavior that an individual utilizes when seeking social support. Item scores of the SSRS were simply added up, generating a total support score ranging from 12 to 66 , a subjective support score ranging from 8 to 32, an objective support score ranging from 1 to 22 , and a support-seeking behavior score ranging from 3 to 12, respectively. The total scores of all these ten items are used to assess the current social support status of individuals. Higher scores indicate stronger social support.

The DHI evaluates the self-perceived handicapping effects of vestibular disease and its impact on quality of life. In this study, The DHI is a questionnaire consists of 25 questions, with seven related to physical aspects (DHI-P), nine related to emotional aspects (DHI-E), and nine related to functional aspects (DHI-F). Each item is assigned 0, 2, or 4 points; therefore, the DHI score is between 0 and 100 points. In this study, the total DHI score and the score for each domain were calculated. The higher the score, the worse the impact of dizziness on the quality of life of the patient; the lower the score, the lower the impact [25].

\section{Demographic and clinical assessment}

Detailed demographic and clinical parameters were recorded on admission. Including age, gender, hypertension (receive antihypertensive treatment or blood pressure $\geq 140 / 90 \mathrm{~mm} \mathrm{Hg}$ on repeated measurements), diabetes mellitus (receive medicine for diabetes mellitus, fasting blood glucose $\geq 126$ $\mathrm{mg} / \mathrm{dl}, 2 \mathrm{~h}$ postprandial blood glucose or random blood glucose $\geq 200 \mathrm{mg} / \mathrm{dl}$ ), current smoking (current or quit smoking $\leq 6$ months prior) and drinking (intake more than $80 \mathrm{~g} / \mathrm{d}$ or quit drinking $\leq 6$ months prior), Systolic Blood Pressure (SBP) and Diastolic Blood Pressure (DBP) on admission and history of ischemic heart disease. 
Social support reduces residual dizziness after canalith repositioning procedure in benign paroxysmal positional vertigo

\section{Statistical analyses}

First, we investigated the difference in baseline characteristics between patients with $\mathrm{RD}$ and those without. Univariate analysis was performed with student's t-tests for continuous variables and Chi-square test for categorical variables. Continuous variables are expressed as means \pm Standard Deviation (SD) and categorical variables are given as the percentage of patients affected. Multiple logistic regression model was performed to determine the independent association between social support and RD after adjusted confounding factors with $\mathrm{P}$ value $\leq 0.1$ in univariate analysis. Relationship between SSRS score and DHI score was tested using the Pearson product-moment correlation. Data were analysed with the SPSS Version 19. For all analyses, a two-tailed value of $\mathrm{p}<0.05$ was deemed as significant.

\section{Results}

\section{Demographic and general characteristics}

Two hundreds and fifty-eight patients were enrolled in this prospective study, comprising 116 men and 142 women aged $52.44 \pm 12.71 \mathrm{y}$. The PC, HC, AC and multiple BPPV were involved in 147, 86, 16 and 9 patients, respectively. The length from BPPV onset to CRPs was $3.95 \pm 2.50 \mathrm{~d}$. Baseline characteristic was presented in Table 1. Of them, 79 (30.62\%) patients had hypertension, 35 (13.57\%) patients had diabetes mellitus, 81 (31.40\%) patients had current smoking and 52 (20.16\%) patients had drinking, 19 (7.36\%) patients had a history of ischemic heart disease.

Table 1. Comparison between patients with RD and those without.

\begin{tabular}{|c|c|c|c|}
\hline & Residual dizziness (N=105) & No residual dizziness $(\mathrm{N}=153)$ & $\mathbf{P}$ \\
\hline Age, y, mean (SD) & $55.30 \pm 12.24$ & $50.48 \pm 12.69$ & 0.003 \\
\hline Female, N (\%) & $66(62.9)$ & $76(49.7)$ & 0.036 \\
\hline Hypertension, N (\%) & $31(29.5)$ & $48(31.4)$ & 0.752 \\
\hline Diabetes mellitus, $\mathrm{N}(\%)$ & $12(11.4)$ & $23(15.0)$ & 0.406 \\
\hline Ischemic heart disease, N (\%) & $8(7.6)$ & $11(7.2)$ & 0.897 \\
\hline Smoking, N (\%) & $36(34.4)$ & $45(29.4)$ & 0.407 \\
\hline Drinking, N (\%) & $24(22.9)$ & $28(18.3)$ & 0.37 \\
\hline BMI, mean (SD) & $24.06 \pm 3.15$ & $23.32 \pm 3.20$ & 0.068 \\
\hline SBP, mmHg, mean (SD) & $141.01 \pm 18.72$ & $142.63 \pm 20.11$ & 0.514 \\
\hline DBP, mmHg, mean (SD) & $83.50 \pm 9.30$ & $85.38 \pm 9.86$ & 0.124 \\
\hline Onset to CRPs, d, mean (SD) & $4.43 \pm 2.52$ & $3.62 \pm 2.44$ & 0.011 \\
\hline Involved canal, N (\%) & & & 0.439 \\
\hline Posterior & $62(59.0)$ & $85(55.6)$ & \\
\hline Horizontal & $31(29.5)$ & $55(35.9)$ & \\
\hline Anterior & $9(8.6)$ & $7(4.6)$ & \\
\hline Multiple & $3(2.9)$ & $6(3.9)$ & \\
\hline \multicolumn{4}{|l|}{ DHI score mean (SD) } \\
\hline Total & $59.18 \pm 18.20$ & $44.46 \pm 14.91$ & 0 \\
\hline DHI-E & $18.32 \pm 7.68$ & $8.44 \pm 5.41$ & 0 \\
\hline DHI-F & $21.52 \pm 7.60$ & $16.88 \pm 7.02$ & 0 \\
\hline DHI-P & $20.13 \pm 6.05$ & $19.14 \pm 7.56$ & 0.261 \\
\hline \multicolumn{4}{|l|}{ SSRS score, mean (SD) } \\
\hline Total & $34.00 \pm 8.54$ & $38.32 \pm 6.48$ & 0 \\
\hline Subject support & $19.67 \pm 6.10$ & $22.49 \pm 5.39$ & 0 \\
\hline Objective support & $7.96 \pm 2.63$ & $8.71 \pm 2.43$ & 0.02 \\
\hline
\end{tabular}


BMI: Body Mass Index; SBP: Systolic Blood Pressure; DBP: Diastolic Blood Pressure; CRP: Canalith Repositioning Procedure; DHI: Dizziness Handicap Inventory; SSRS: Social Support Rating Scale.

\section{Factors associated with $R D$}

One hundred and five $(40.70 \%)$ patients complained of RD after successful CRP. The duration of RD was $17.92 \pm 12.75 \mathrm{~d}$ (range $=4-74 \mathrm{~d}$, median $=15 \mathrm{~d}$ ). Univariate analysis indicated that the age $(\mathrm{P}=0.003)$, the proportions of female sex $(\mathrm{P}=0.036)$, the length from BPPV onset to CRPs $(\mathrm{P}=0.011)$ and DHI total score $(\mathrm{P}=0.000)$ were significantly higher in patients with RD compared with those without. The SSRS total score $(\mathrm{P}=0.000)$, on the contrary, tended to be lower in patients with RD. The scores derived from the 3 subdomains of SSRS were also compared. We observed a significant difference between patients who experienced RD and those who did not develop this disorder accounting for the objective support $(\mathrm{P}=0.020)$, subjective support $(\mathrm{P}=0.000)$ and the degree of social utilization ( $\mathrm{P}=0.002)$. After adjusting the confounding factors, multivariate logistic regression analysis suggested that the SSRS total score (OR 0.923, 95\% CI 0.889-0.958, $\mathrm{P}=0.000$ ) and the 3 subdomains of SSRS (low subject support (OR $0.912,95 \%$ CI $0.869-0.956, \mathrm{P}=0.000)$, objective support (OR $0.889,95 \%$ CI $0.799-0.989, \mathrm{P}=0.031)$, and degree of social utilization (OR 0.825, 95\% CI 0.716-0.951, P=0.008) ) were also independently associated with the presence of RD after CRPs. In addition, a negative correlation was found between SSRS total score and the duration of RD ( $r=-0.83, \mathrm{P}=0.000$, Table 2).

Table 2. Independent association between social support and RD based on multiple logistic regression analysis.

\begin{tabular}{|c|c|c|c|c|c|c|}
\hline & \multicolumn{3}{|l|}{ Crude } & \multicolumn{3}{|c|}{ Adjusted $^{\dagger}$} \\
\hline & OR & $95 \% \mathrm{Cl}$ & $\mathbf{P}$ & OR & $95 \% \mathrm{Cl}$ & $\mathbf{P}$ \\
\hline \multicolumn{7}{|l|}{ SSRS score } \\
\hline Total score & 0.926 & 0.894-0.959 & 0 & 0.923 & $0.889-0.958$ & 0 \\
\hline \multicolumn{7}{|l|}{ The 3 subdomains } \\
\hline Subject support & 0.918 & $0.878-0.960$ & 0 & 0.912 & $0.869-0.956$ & 0 \\
\hline Objective support, & 0.887 & $0.801-0.983$ & 0.022 & 0.889 & $0.799-0.989$ & 0.031 \\
\hline Degree of social utilization & 0.812 & $0.708-0.931$ & 0.003 & 0.825 & $0.716-0.951$ & 0.008 \\
\hline
\end{tabular}

${ }^{\dagger}$ Adjustment by Age, Female, BMI, Onset to CRPs.

\section{Discussion}

One hundred and five (40.70\%) patients with BPPV occurred RD after successful repositioning in our study, the rate of which was similar to that in previous studies that ranged from $36.6 \%$ and $61 \%$, which might be explained by diverse diagnostic criteria for RD and the different inclusion criteria in these trials [8-10].

RD has never been considered a disease entity with its own features but rather the short and unpleasant side effects of the earlier BPPV because of the benignity and transience of symptoms. Pathophysiology of RD is yet incompletely understood. Several possible mechanisms have been proposed to explain the occurrence of residual symptoms after a successful treatment for BPPV. First, the persistence of otoconial debris due to incomplete repositioning could produce mild positional vertigo, where the remaining debris is insufficient to provoke noticeable positional nystagmus; Second, BPPV is not only a disorder of the semicircular canals but also a disorder of the otoliths, and otolith dysfunction might account for transient dizziness; Third, another vestibular lesion which is difficult to identify from the history and clinic examination might coexist with BPPV; fourth, delayed recovery might be due to the longer time needed for central adaptation after particle repositioning; These hypotheses, although theoretically valid, have not yet been supported by definitive data [5-10].

It is widely understood that social support is a network of family, friends, neighbors, colleagues or anyone a person can turn to when faced with some trouble. Currently, the impact of social support on the recovery from prior disease has been a research focus and concerned by scholars. Previous studies had reported that there were certain association between social support and rehabilitation [16,17]. The good social support had been considered as essential strength and encouragement which can excite people to take vigorous action and overcome difficulties. In this study, we not only evaluated total SSRS score but also considered the 3 items score of SSRS between patients with RD and those without. Results suggested that good social support have a favourable impact on BPPV recovery, which further support the positive effects of social support on healing process. 
The objective support reflects the degree of actual support that an individual received in the past. The subjective support refers to emotional experience and reflects the perceived interpersonal network that an individual can count on. The support utilization refers to the pattern of behavior that an individual utilizes when seeking social support. It is well known that anxiety and dizziness are comorbid symptom, and anxiety has been demonstrated to play a contributory role in dizziness $[1-3,26]$. In this study, for patients with $\mathrm{RD}$, the ability to deal with daily work and interact with others was impaired because of the functional limitation in daily living, and the feelings of insecurity, anxiety, and depression experienced by patients as result of their disorders. That was to say, the ability to seek support from others was impaired. In addition, it was reported that lack of subjective and objective support will bring negative emotion, such as nervousness, worry, fear, sadness and despair, which ratcheted up the anxiety and depression even further. It is well known that the love and care from our family bring us warmth, however, RD has a great impact on the quality of life of patients and their family caregivers that provide long-term day-to-day care. Both the impaired ability to interact with others and the negative emotion could taxes the energy and patience of their family members, which might further lead to a low social support. In addition, low utilization of support, further lead to social support can't be fully utilized. In all, the low social support and utilization of support could cause decreased psychological endurance and the capacity to deal with troubles.

The DHI is a survey that includes functional, emotional, and physical effects of dizziness on an individual. It can be used for many scholars to assess the residual symptoms and its impact on the patient's social and emotional life after CRP [27,28]. In this study, a statistically significant difference in the DHI total score and the 3 subdomains of DHI was found between patients who developed $\mathrm{RD}$ and those free from residual symptoms after successful CRP, which was same with previous studies. Patients with RD had a greater decrease in the quality of life. In addition, a negative correlation between SSRS total score and DHI total score $(\mathrm{r}=-0.80, \mathrm{P}=0.000)$ was found in this study, which further proved the importance of social support in the recovery of BPPV.

Improving microcirculation vestibular rehabilitation and drugs had been proposed as possible treatment of residual symptoms after BPPV. However, the effectiveness need to be further confirmed in randomized controlled trials [19]. In this study, BPPV patients with good social support suffered less RD after CRP and had a better quality of social and emotional life suggested by the score of DHI. For patients with BPPV, we should evaluated the amount of social support and provide constructive guidance in daily life. It was necessary to offer psychological intervention and to distract patient's attention from the bad residual experience by participating in social activities and interacting with others. The ultimate goal was to help patients seeking social support actively, which was important to the patients' recovery and their daily life.
In conclusion, residual dizziness is a common condition in patients with BPPV after CRP. However, neither evidencebased nor consensus-based guidelines exist recommending how to deal with RD because of the unknown etiology. Our study indicates that social support could help in reducing the incidence of RD in patients with BPPV after successfully CRP and returning to daily life more rapidly and comfortably.

\section{Acknowledgement}

All the authors listed have approved the submitted manuscript and we declare that we have no conflict of interest.

\section{References}

1. Lee SH, Kim JS. Benign paroxysmal positional vertigo. J Clin Neurol. 2010; 6: 51-63.

2. Hornibrook J. Benign Paroxysmal Positional Vertigo (BPPV): history, pathophysiology, office treatment and future directions. Int J Otolaryngol 2011; 2011: 835671.

3. Martellucci S, Pagliuca G, de Vincentiis M. Features of residual dizziness after canalith repositioning procedures for benign paroxysmal positional vertigo. Otolaryngol Head Neck Surg 2016; 154: 693-701.

4. Martens C, Goplen FK, Nordfalk KF, Aasen T, Nordahl $\mathrm{SH}$. Prevalence and characteristics of positional nystagmus in normal subjects. Otolaryngol Head Neck Surg 2016; 154: 861-867.

5. Faralli M, Cipriani L, Del Zompo MR, Panichi R, Calzolaro L, Ricci G. Benign paroxysmal positional vertigo and migraine: analysis of 186 cases. B-ENT 2014; 10: 133-139.

6. van der Scheer-Horst ES, van Benthem PP, Bruintjes TD, van Leeuwen RB, van der Zaag-Loonen HJ. The efficacy of vestibular rehabilitation in patients with benign paroxysmal positional vertigo: a rapid review. Otolaryngol Head Neck Surg 2014; 151: 740-745.

7. Balatsouras DG, Aspris A, Ganelis P, Koukoutsis G, Moukos A, Fassolis A. Duration of benign paroxysmal positional vertigo as a predictor for therapy. B-ENT 2015; 11: 199-203.

8. Jung Im Seok, Hyo Min Lee, Ji Hoon Yoo. Residual dizziness after successful repositioning treatment in patients with benign paroxysmal positional vertigo. J Clin Neurol 2008; 4: 107-110.

9. Hyun-Ah Kim, Hyung Lee. Autonomic dysfunction as a possible cause of residual dizziness after successful treatment in benign paroxysmal positional vertigo. Clinical Neurophysiology 2014; 125 608-614.

10. Acar B, Karasen RM, Buran Y. Efficacy of medical therapy in the prevention of residual dizziness after successful repositioning maneuvers for Benign Paroxysmal Positional Vertigo (BPPV). B-ENT 2015; 11: 117-121.

11. Tang WK, Chen YK, Lu JY, Chu WC, Mok VC, Ungvari GS, Wong KS. Cerebral microbleeds and depression in lacunar stroke. Stroke 2011; 42: 2443-2446. 
12. Du H, King RB, Chu SK. Hope, social support, and depression among Hong Kong youth: personal and relational self-esteem as mediators. Psychol Health Med 2016; 21: 926-931.

13. Roberts CA, Geryk LL, Sage AJ. Adolescent, caregiver, and friend preferences for integrating social support and communication features into an asthma elf-management app. J Asthma 2016; 53: 948-954.

14. Marcin R, Wlodzimierz O, Katarzyna S. Pain intensity, temperament traits and social support as determinants of trauma symptoms in patients suffering from rheumatoid arthritis and low-back pain. Int J Rheum Dis 2016; 19: 412-419.

15. Maria GM, Carlos C, Giovanni L. Social support, socioeconomic status, health and abuse among older people in seven European countries. PLoS One 2013; 8: 54856.

16. Emily M Bucholz, Kelly M Strait, Rachel P Dreyer. Effect of low perceived social support on health outcomes in young patients with acute myocardial infarction: results from the VIRGO (Variation in Recovery: Role of Gender on Outcomes of Young AMI Patients) Study. J Am Heart Assoc 2014; 3: 001252.

17. Christine L, Sonia S, Judith WR. Perceived social support, coping styles, and Chinese immigrants cardiovascular responses to stress. Int J Behav Med 2012; 19: 174-185.

18. Salvatore M, Giulio P, Marco de V. Features of residual dizziness after canalith repositioning procedures for benign paroxysmal positional vertigo. Otolaryngol Head Neck Surgery 2016; 154: 693-701.

19. Bhattacharyya N, Baugh RF, Orvidas L. American Academy of Otolaryngology-Head and Neck Surgery Foundation. Clinical practice guideline: benign paroxysmal positional vertigo. Otolaryngol Head Neck Surg 2008; 139: 47-81.

20. Wegner I, Niesten ME, van Werkhoven CH, Grolman W. Rapid systematic review of the epley maneuver versus vestibular rehabilitation for benign paroxysmal positional vertigo. Otolaryngol Head Neck Surg 2014; 151: 201-207.
21. Lempert T, Tiel-Wilck K. A positional maneuver for treatment of horizontal-canal benign positional vertigo. Laryngoscope 1996; 106: 476-478.

22. Nuti D, Agus G, Barbieri MT. The management of horizontal-canal paroxysmal positional vertigo. Acta Otolaryngol 1998; 118: 455-460.

23. Xu J, Wei Y. Social support as a moderator of the relationship between anxiety and depression: an empirical study with adult survivors of Wenchuan earthquake. PLoS One 2013; 8: 79045.

24. Feng S, Tan H, Benjamin A. Social support and posttraumatic stress disorder among flood victims in Hunan, China. Ann Epidemiol 2007; 17: 827-833.

25. Chen W, Shu L, Wang Q. Validation of 5-item and 2-item questionnaires in Chinese version of dizziness handicap inventory for screening objective benign paroxysmal positional vertigo. Neurol Sci 2016; 37: 1241-1246.

26. Steffi W, Annette BB. Health-related quality of life and emotional distress in patients with dizziness: a crosssectional approach to disentangle their relationship. BMC Health Serv Res 2014; 14: 317.

27. Jacobson GP, Newman CW, Hunter L, Balzer GK. Balance function test correlates of the dizziness handicap inventory. J Am Acad Audiol 1991; 2: 253-260.

28. Whitney SL, Marchetti GF, Morris LO. Usefulness of the dizziness handicap inventory in the screening for benign paroxysmal positional vertigo. Otol Neurotol 2005; 26: 1027-1033.

\section{*Correspondence to}

Xinjiang Zhang

Department of Neurology

The First People's Hospital of Yangzhou

PR China 